\title{
A Review of Nanoscale Channel and Gate Engineered FINFETs for VLSI Mixed Signal Applications Using Zirconium-di-Oxide Dielectrics
}

\author{
D.Nirmal $^{*}{ }^{* 1}$, P.Vijaya Kumar ${ }^{2}$, K. Shruti ${ }^{1}$, Binola K Jebalin ${ }^{1}$ and N.Mohankumar ${ }^{3}$ \\ ${ }^{1}$ Department of Electronics and Communication Engineering, Karunya University, Coimbatore, Tamilnadu, India \\ ${ }^{2}$ Department of Electrical and Electronic Engineering, Karpagam College of Engineering, Coimbatore, Tamilnadu, India \\ ${ }^{3}$ Department of Electronics and Communication Engineering, Skp Engineering College, Thiruvanamalai Tamilnadu, India
}

Received 1 June 2014; Accepted 16 July 2014

\begin{abstract}
In the past, most of the research and development efforts in the area of CMOS and IC's are oriented towards reducing the power and increasing the gain of the circuits. While focusing the attention on low power and high gain in the device, the materials of the device also been taken into consideration. In the present technology, Computationally intensive devices with low power dissipation and high gain are becoming a critical application domain. Several factors have contributed to this paradigm shift. The primary driving factor being the increase in scale of integration, the chip has to accommodate smaller and faster transistors than their predecessors. During the last decade semiconductor technology has been led by conventional scaling. Scaling, has been aimed towards higher speed, lower power and higher density of the semiconductor devices. However, as scaling approached its physical limits, it has become more difficult and challenging for fabrication industry. Therefore, tremendous research has been carried out to investigate the alternatives, and this led to the introduction of new Nano materials and concepts to overcome the difficulties in the device fabrications. In order to reduce the leakage current and parasitic capacitance in devices, gate oxide high-k dielectric materials are explored. Among the different high-k materials available the nano size Zirconium dioxide material is suggested as an alternate gate oxide material for devices due to its thermal stability and small grain size of material. To meet the requirements of ITRS roadmap 2012, the Multi gate devices are considered to be one of the most promising technologies for the future microelectronics industry due to its excellent immunity to short channel effects and high value of On current. The double gate or multi gate devices provide a better scalability option due to its excellent immunity to short-channel effects. Here the different high-k materials are replaced in different Multi Gate MOSFET devices and its performance were studied.
\end{abstract}

Keywords: Lateral Asymmetric Channel, carrier transport efficiency, System-on-chip (SoC), Equivalent Oxide Thickness (EOT), Threshold voltage roll-off.

\section{Motivation to use high $k$ material}

In semiconductor industry Moore's Law clearly stated that we will be compressing twice the number of transistors in every 18 months [1]-[3]. The 2012 ITRS(International Technology Roadmap for Semiconductor) update clearly explained that we are progressing towards different high $\mathrm{k}$ dielectric materials that will soon replace the gate oxide $\mathrm{SiO}_{2}$ of the devices [4]. As stated in the ITRS (2012 Edition), "The gate dielectric has emerged as one of the most difficult challenges for future device scaling". Indeed, the conventional gate dielectric $\mathrm{SiO}_{2}$ obviously cannot survive the challenge of an EOT(equivalent oxide thickness) $=1 \mathrm{~nm}$. Also no manufacturable solutions have yet been found to fabricate the $\mathrm{SiO}_{2}$ thickness, as projected by the ITRS, so it is highly preferable that materials with high dielectric constants and high physical thicknesses will be used for devices [5]. According to Equation. 1, if a dielectric material with a high dielectric constant (high-k) can replace $\mathrm{SiO}_{2}(\mathrm{k}=$ 3.9), the dielectric layer thickness can be increased proportionally while keeping the same capacitance $\mathrm{C}_{\mathrm{ox}}$. A

\footnotetext{
*E-mail address: dnirmalphd@gmail.com ISSN: $1791-2377 @ 2014$ Kavala Institute of Technology. All rights reserved.
}

figure of merit to judge a high $\mathrm{k}$ gate dielectric layer is the EOT, defined as,

$$
\mathrm{EOT}=\left(\mathrm{k}_{\mathrm{sio} 2} / \mathrm{k}_{\text {high }-\mathrm{k}}\right) \mathrm{d}_{\text {high-k }}[6]
$$

The EOT shows the electrical equivalent thickness of the high-k layer to $\mathrm{SiO}_{2}$ when the capacitance is the same. However, before a new high-k material can be integrated into the present ULSI process flow, many requirements have to be met first [7-10]. One of the most crucial elements that allow the successful scaling in the past is certainly the electrical properties of $\mathrm{SiO}_{2}$ [7-9]. First, it can be thermally grown on $\mathrm{Si}$ with excellent control of thickness and uniformity, which forms a very stable interface on the $\mathrm{Si}$ substrate with a low defect density. $\mathrm{SiO}_{2}$ is thermally stable up to $1000^{\circ} \mathrm{C}$, which is required for the MOSFET fabrication. The band gap of $\mathrm{SiO}_{2}$ is large, i.e., $\sim 9 \mathrm{eV}$, with sufficiently large conduction and valence band offsets. The dielectric breakdown field is $\sim 13 \mathrm{MV} / \mathrm{cm}$. In addition, $\mathrm{SiO}_{2}$ is water insoluble, which facilitates photolithography. The use of a polysilicon (Poly-Si) gate electrode in the selfaligned CMOS technology was also a determining factor in the scaling. All the key parameters selected gate dielectric materials related to device is listed in Table.1. 
Table 1 List of Key parameters of selected gate dielectric materials. [6, 11, 12]

\begin{tabular}{llllll}
\hline & $\begin{array}{l}\text { Dielectric } \\
\text { Constant } \\
(\mathbf{( k )}\end{array}$ & $\begin{array}{l}\text { Band } \\
\mathbf{G a p} \\
(\mathbf{E g}, \text { in } \\
\mathbf{e V})\end{array}$ & $\begin{array}{l}\text { Electron } \\
\text { Offset } \\
(\mathbf{\Phi B e}, \text { in } \\
\mathbf{e V})\end{array}$ & $\begin{array}{l}\text { Hole } \\
\text { Offset } \\
(\mathbf{\Phi B p}, \\
\text { in eV) }\end{array}$ & $\begin{array}{l}\text { Breakdown } \\
\text { Field } \\
(\mathbf{E B D}, \text { in } \\
\mathbf{M V} / \mathbf{c m})\end{array}$ \\
\hline $\mathrm{SiO}_{2}$ & 3.9 & 9 & 3.5 & 4 & 10 \\
$\mathrm{Al}_{2} \mathrm{O}_{3}$ & 8 & 8.8 & 3 & 4.7 & 10 \\
$\mathrm{TiO}_{2}$ & 80 & 3.5 & 1.1 & 1.3 & 3 \\
$\mathrm{ZrO}_{2}$ & 25 & 5.8 & 1.4 & 3.3 & $4-5$ \\
$\mathrm{HfO}_{2}$ & 25 & 6 & 1.5 & 3.4 & $4-5$ \\
\hline
\end{tabular}

\section{Selection of Zirconium as high $\mathrm{k}$ material}

There have been many review papers, and industry consensus that proposed a criterion for choosing alternative high-k materials for in MOSFET for different applications. [ $8,9,11,12,13,14]$

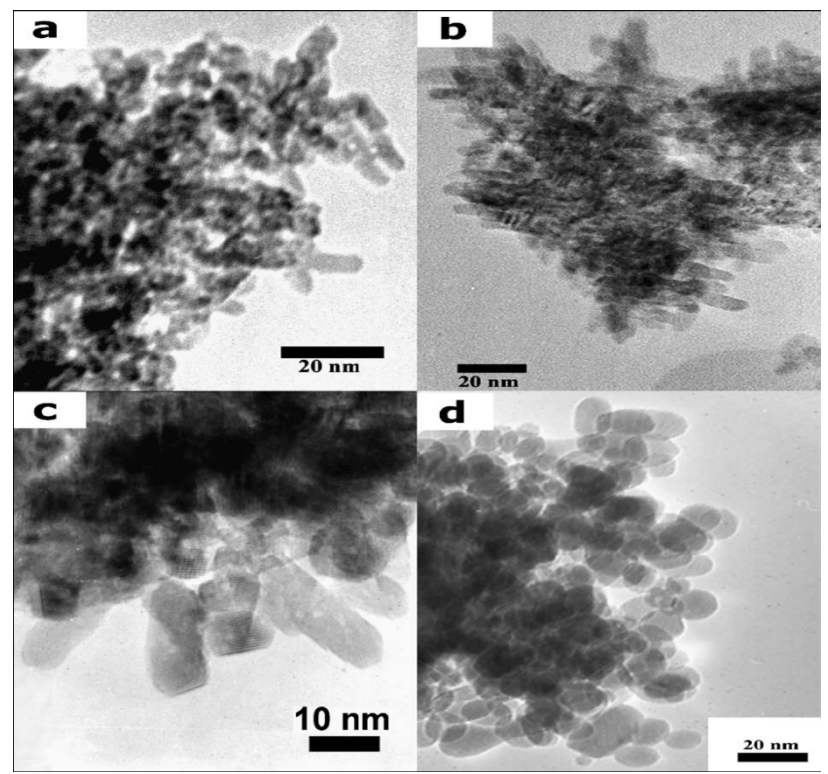

Fig. 1. TEM microphotograph of nanocrystalline $\mathrm{ZrO}_{2}$ particles prepared by hydrothermal method: a- c - high-temperature hydrolysis of $0.25 \mathrm{M}$ aqueous $\mathrm{ZrO}\left(\mathrm{NO}_{3}\right) 2$ solutions $(\mathrm{a}-(\mathrm{T}=150 \mathrm{jC}, \mathrm{s}=10 \mathrm{~min})$; $\mathrm{b}-(\mathrm{T}=250 \mathrm{jC}, \mathrm{s}=10 \mathrm{~min}) ; \mathrm{c}-(\mathrm{T}=250 \mathrm{jC}, \mathrm{s}=6 \mathrm{~h})) ;(\mathrm{d})$

hydrothermal treatment of amorphous gel of $\mathrm{ZrO}_{2} \cdot \mathrm{nH}_{2} \mathrm{O}(\mathrm{T}=250 \mathrm{jC}, \mathrm{s}$ $=6 \mathrm{~h})$. [15]

Its observed that Zirconiam-di-oxide is one of the alternate material for gate oxide in the FinFET[75]. Zirconia is the most appropriate metal oxide since it is thermodynamically stable with $\mathrm{Si}[15]$. The figure.1 shows the TEM microphotograph of nanocrystalline $\mathrm{ZrO}_{2}$ particles prepared by hydrothermal method and stability of the material in the nanosize, since it has a dielectric constant value of 25 , a large energy bandgap it is suggested for FinFET device. (With values reported from 5.16 to $7.8 \mathrm{eV}$ ) $[16,17]$.

\section{Preparation of Zirconium Dioxide}

\subsection{Dry chemical process}

The wet chemical method is employed for the preparation of $\mathrm{ZrO}_{2}$ since the powders with different particle size, morphology and phase composition can be prepared by varying parameters such as temperature, duration of the processing, concentration of chemical species to solution.

The zirconia nano particles were obtained by the refinement of zirconium dioxide. The Chemical reactions are as follows[75].

$$
\begin{aligned}
& \mathrm{ZrO}_{2}+4 \mathrm{HNO}_{3} \rightarrow \mathrm{Zr}\left(\mathrm{NO}_{3}\right)_{4}+2 \mathrm{H}_{2} \\
& 2 \mathrm{Zr}\left(\mathrm{NO}_{3}\right)_{4}+2 \mathrm{Na}\left(\mathrm{NO}_{3}\right) \rightarrow 2 \mathrm{NaZrO}_{2}+5 \mathrm{~N}_{2}+13 \mathrm{O}_{2} \\
& 2 \mathrm{NaZrO}_{2}+2 \mathrm{HCl} \rightarrow 2 \mathrm{ZrO}_{2}+2 \mathrm{NaCl}+\mathrm{H}_{2}
\end{aligned}
$$

The zirconium dioxide (99.9\%-AR grade) was purchased from Himedia chemicals. All other chemical reagents in the experiments were of analytical grade and the water used was deionised. Typically the mixed solution of zirconium dioxide $(0.04 \mathrm{M})$ and $\mathrm{HNO}_{3}(20 \mathrm{ml})$, with appropriate gram molecular weight, gives the highly hygroscopic zirconium (IV) nitrate salt which is made to be a solution immediately. The solution of Zirconium (IV) nitrate was rapidly mixed with sodium nitrate. The precipitate was filtered and washed with dilute $\mathrm{HCl}$ and demonized water repeatedly. Then the precipitate was dried at controlled atmosphere at $100^{\circ} \mathrm{C}[75]$. The outcome of this preparation given the thermally stable with nanosize Zirconium-di-oxide material is observed[75], it gives excellent improvement in the FinFET devices.

\section{Limits Of CMOS Technology}

Since the 1970s people have been predicting the end of CMOS [18]. Despite these predictions, the monetary benefit of growth has driven massive research, which has overcome all previous barriers [19]. However, many experts are now claiming that the industry is reaching limits that no amount of research can push past. The scaling of CMOS has resulted in a strong improvement in the RF performance of MOS devices [20]. In order to improve device performance, we have to search for a breakthrough in those area involving device structures, material selection, fabrication technology and device operating mechanism to solve problems existing in power, integration density, property optimization and process [21].

There are three key factors limiting continued scaling in CMOS:

- Minimum dimensions that can be fabricated

- Diminishing returns in switching performance

- Off-state leakage

So far the primary limitations to chip scaling have been lithographic issues. In lithographic the research is being done to reduce the minimum wavelength into the extreme ultraviolet spectrum (13 nm wavelengths) [22]. However, transistor dimensions are approaching a physical limit that cannot be overcome. That limit is the size of the atom and molecule of the material. Clearly devices cannot be fabricated smaller than the dimension of a single molecule and some dimensions will need to be more than a molecule wide. It should also be pointed out here that lithographic equipment costs have also grown exponentially [23], and this is beginning to limit the profitability of increased scaling. The dominant non-ideal effect that must be addressed for current scaling to continue is off-state power consumption. Within digital logic, the sources that 
contribute to off-state power consumption are: junction leakage, gate induced drain leakage, sub-threshold channel current, and gate tunnel currents. In fact, leakage currents grow exponentially as gate length decreases [24]. To reduce this limits let us move on to the solutions the researchers are proposed.

\section{Study of various logic styles and reduce the power:}

\section{SILICON-ON-NOTHING}

Silicon-On-Nothing transistors with gate length varying from $0.25 \mathrm{um}$ down to $80 \mathrm{~nm}$ exhibit excellent performance and scalability. The silicon-on-nothing architecture with thin fully depleted Si film and ultra thin buried oxide results in attenuated short-channel effects, high ON current and high electron mobility[25,28]. The proposed model reproduces the variations of the threshold voltage and sub threshold swing and it is leads for further device optimization.

\section{WAFER BONDING}

Wafer bonding is an enabling technology that allows the fabrication of a variety of complicated structures that would be difficult or impossible to make by other means. The process of hydrophobic bonding removes the thin native oxide at surface of silicon wafers. For devices that utilize bulk conduction through the bond interface, this native oxide would reduce or destroy performance by creating traps and electrical discontinuities that will affect the $\mathrm{I}-\mathrm{V}$ and $\mathrm{C}-\mathrm{V}$ characteristics at the interface[25,28].

\section{FinFETs}

FinFETs have emerged as a superior alternative to replace the conventional bulk MOSFETs to continue the scaling. The distinguishing characteristic of the FinFET is that the conducting channel is wrapped around a thin silicon "fin", which forms the body of the device. The dimensions of the fin determine the effective channel length of the device even down to a 10-nm feature size[25]-[30].

In the literature, FinFET is used generically to describe any fin-based multigate MOSFET architecture regardless of number of gates.

\section{Selection Of Finfet}

According to the ITRS 2012 [33], MOS transistors will have gate lengths of around $10 \mathrm{~nm}$ in 2015.The performance of these transistors is expected to be severely degraded by short-channel effects (SCEs). For sub-100 nm scaling of MOSFETs, double-gate field-effect transistors like FinFETs have attracted considerable attention due to their immunity to Short channel effects and proximity to standard bulk planar CMOS processing [31- 36].

FinFET have been proposed to improve the scalability of the MOSFET, for CMOS technology generations beyond the 22-nm technology node. Since an ultrathin silicon body can effectively suppress SCE, a lightly doped or undoped body can be used in order to achieve high carrier mobilities for improved transistor drive current, as well as to minimize variations in threshold voltage due to statistical doping variations [37]-[38]. So the range of work functions required for thin-body MOSFETs is $4.4 \mathrm{eV}-5.0 \mathrm{eV}$ [39], ruling out polycrystalline silicon as a gate material [40].Since the advanced double-gate FinFETs require ultrathin gate dielectrics for low power consumption. Recently, in FinFET structures with ultra-thin $\mathrm{HfO}_{2}$ and $\mathrm{SiON}$ as gate dielectrics, the gate tunnelling current has been investigated experimentally with the source, drain and substrate electrodes grounded [41],[77]. The reported experimental results provide evidence for reduction of the gate tunnelling current density in narrow FinFET structures compared to their counterpart quasi-planar structures [41].

\subsection{Channel and gate engineered FinFETs}

FinFET devices shows considerable threshold voltage roll off and DIBL effects. This adverse threshold voltage roll-off effect is the most daunting road block in future MOSFET design. The minimum acceptable channel length is primarily determined by this roll-off [42]-[43],[76]. So here we introduce channel and gate engineering techniques into the FinFETs to reduce subthreshold leakage current and SCE.

In the channel engineering technique, channel is highly doped near the source region to reduce the width of the depletion region in the vicinity of this junction that resulting in reduced subthreshold leakage current and increased output impedance [44]-[45]. This method is called lateral channel engineering, e.g. halo or pocket implants and the engineered channel is known as Lateral Asymmetric Channel (LAC) or Graded channel $\{\mathrm{GC}\}$. The GC MOSFET is an asymmetric channel device which minimizes the inherent bipolar effects in Fully Depleted transistors [46]. In single halo, an undoped region is preserved in the drain side of the channel. Such undoped region presents negative threshold voltage and can be considered as an extension of the drain region below the gate [47]. Halo implanted devices show excellent output characteristics with low DIBL, higher drive currents, flatter saturation characteristics, and slightly higher breakdown voltages compared to the conventional MOSFET [48]-[49].

In the year 1999 Long et al. [50] proposed a gate engineering technique in which two different materials having different work functions are merged together to form a single gate of a bulk MOSFET. In the DMG structure, the work function of the gate material (M1) close to the source is chosen higher than that close to the drain end (M2) for nchannel FinFETs [51]-[55]. As a result, the electric field and electron velocity along the channel suddenly increase near the interface of the two gate materials, resulting in increased gate transport efficiency, which implies that the threshold voltage under gate material M1 is higher than that under gate material M2. When the drain voltage exceeds the drain saturation voltage, the excess voltage is absorbed by gate metal M2, preventing the drain field from penetrating into the channel. This so-called gate work function engineering allows the DMG devices to have the same threshold voltage for a reduced doping concentration in the channel region, resulting in better immunity to mobility degradation and also higher transconductance [56].

Fig. 4 shows the 2D cross-sectional view of a FinFET. In the FinFET, the gate work function is fixed at $4.577 \mathrm{eV}$ to obtain the threshold voltage of $0.357 \mathrm{~V}$ at a drain voltage of 0.1 V. In Dual Material FinFET,(Fig.4) the work functions of metals M1 (Molybdenum) and M2 (Aluminium) are taken as 4.55 and $4.1 \mathrm{eV}$, respectively, with equal lengths of $\mathrm{L} 1$ and L2, and a threshold voltage of $0.3 \mathrm{~V}$ at a drain voltage of $0.1 \mathrm{~V}$ is obtained. Optimization of the length and concentration of the halo-doped region for the Single Halo FinFET(Fig.4) was carried out, and the optimum length was found to be $20 \mathrm{~nm}$ with a pocket implantation of $7.91 \times 10^{17}$ $\mathrm{cm}^{-3}[78]$. Increase in the halo doping concentration results in reduced short channel effects but on the other hand results 
in increased mobility degradation at the source end and threshold voltage.

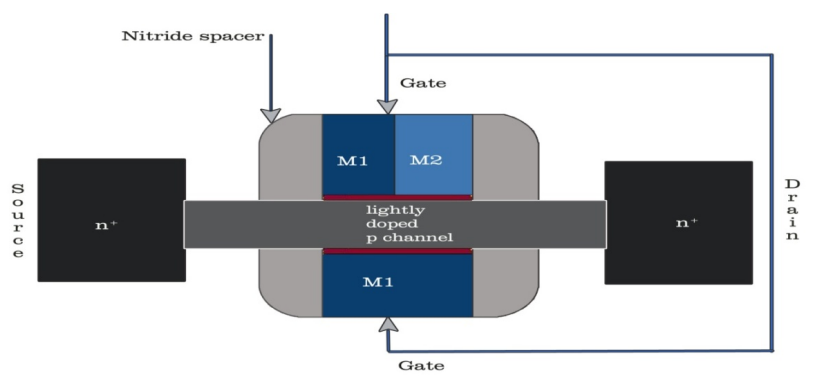

Fig 2. Cross-sectional view of the Dual Material FINFET

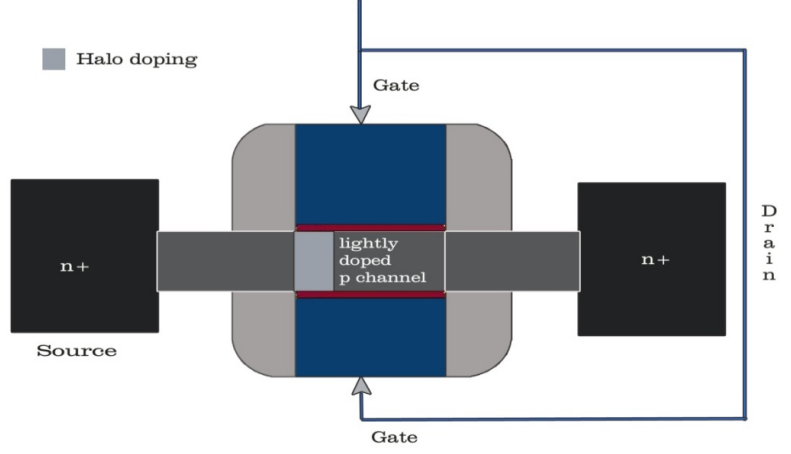

Fig. 3. Cross-sectional view of the Single Halo-FinFET

The leakage current in the channel engineered and gate engineered devices are lesser than the conventional FinFET because of the screening of the drain bias by the step function in the surface potential profile. So any increase in leakage current is suppressed. Gain and output resistance are also higher for these devices. So the Single Halo FinFETs and Dual Material FinFETs can be used for lowpower subthreshold analog applications.

\section{Novel approach to Dielectrics in FINFET}

Since the advent of the metal-oxide semiconductor system over 40 years ago, the $\mathrm{SiO}_{2}$ gate oxide has been serving as the key enabling material in scaling silicon CMOS technology .With the continuous miniaturization of devices, in order to improve the capability of gate control and control the short channel effect, the gate dielectric thickness should be scaled down with channel length. Facing these challenges, scientists have already proposed some applicable solutions, including new materials, new technologies and device structure innovation. The major advances in Nanosize materials with high-k gate dielectric and metal gate to solve the problems existing in the gate stack and the low power issue [57]-[61]. With the integration of high-k dielectrics into FinFET, the performance of the device is further enhanced. The gate oxide material $\left(\mathrm{SiO}_{2}\right)$ in the channel and gate engineered devices are replaced with various high-k dielectrics and the performance of the device is also improved [62]-[63],[79]. The leakage current of the devices are decreased exponentially for high dielectric material which avoids the direct tunnelling of electrons through the insulator. The DIBL which is an important indicator of performance of the devices also decreases exponentially for high dielectric materials thereby suppressing Short Channel Effects of the Nano devices[63]-[64]. The transconductance of the devices also increases with $\mathrm{k}$ value. Gain and output resistance of the devices are expected to increase linearly with $\mathrm{k}$ value. The most optimized results are can be obtained for devices with $\mathrm{ZrO}_{2}$ as the gate dielectric. Thus the integration of $\mathrm{ZrO}_{2}$ nano material dielectrics will improved the performance emerging novel FINFET device.

\section{Summary}

Most predictions are, if trends continue, CMOS scaling can continue for only a decade or two [65],[66],[67]. There have been many review papers and industry consensus that proposed criteria for choosing alternative high-k materials for different applications [66]-[74]. From the various analysis and investigations of these literatures, Zirconium dioxide was found out to be the best alternative for $\mathrm{SiO}_{2}$ because of its excellent thermal stability, capacitive performance and insulating properties. So Zirconium dioxide as dielectric nano materials in transistors in the future of nanotechnology. These transistors are expected to show excellent performance and they also show reduce shortchannel effects (SCEs). For sub-100 nm scaling of MOSFETs, double-gate field-effect transistors like FinFETs have attracted considerable attention due to their immunity to SCEs and proximity to standard bulk planar CMOS processing. Furthermore the gate and channel engineering techniques employed in FinFETs enhance the device performance to a great extend. These techniques suppress the Short Channel Effects such as DIBL in the FinFETs and also reduces the leakage current. The transconductance and output resistance of the FinFETs increases. The integration of $\mathrm{ZrO}_{2}$ as gate dielectric in these channel and gate engineered FinFETs further suppresses the leakage current and Short Channel effects making them more applicable for low power subthreshold analog performance.Thus the channel and gate engineered FinFETs with $\mathrm{ZrO}_{2}$ are promising candidates for the future semiconductor devices for VLSI systems.
1. G. E. Moore, Electron., 38, 114 (1965).

2. Silicon Moore's Law, Intel ${ }^{\circledR}$ website, http://www.intel.com/research/silicon/mooreslaw.htm (Retrieved July 2004).

3. Novel materials for future transistor generations, Gennadi Bersuker , Howard R. Huff International SEMATECH, Austin, TX 78741, USA.

4. http://www.itrs.net/Links/2012ITRS/Home2012.html

5. Hasanur R. Khan, Denis Mamaluy, Dragica Vasileska (2008),"Approaching Optimal Characteristics of 10-nm HighPerformance Devices: A Quantum Transport Simulation Study of Si FinFET", IEEE transactions on Electron Devices, vol. 55, no. 3, pp.743-753.

\section{References}

6. D.J.Hubbard, and D.G., "Thermodynamic stability of binary oxides in contact with Silicone", J.mater.Res.11(11)2757(1996)

7. T. Hori, "Gate Dielectrics and MOS ULSIs: Principles, Technologies, and Applications, "Springer, Berlin, Germany (1997).

8. M. Houssa, High-k Gate Dielectrics, Institute of Physics Publishing, Bristol, UK (2004).

9. H. R. Huff, A. Hou, C. Lim, Y. Kim, J. Barnett, G. Bersuker, G. A. Brown, C. D. Young, P. M. Zeitzoff, and J. Gutt, Microelectron. Eng., 69, 152 (2003).

10. International Technology Roadmap for Semiconductors, Semiconductor Industry Association, San Jose, CA (2010). 
11. L. Manchanda, M. D. Morris, M. L. Green, R. B. van Dover, F. Klemens, T. W. Sorsch, P. J. Silverman, G. D. Wilk, B. Busch, and S. Aravamudhan, Microelectron. Eng., 59, 351 (2001).

12. J. Robertson, Appl. Surf. Sci., 190, 2 (2002).

13. G. D. Wilk, R. M. Wallace, and J. M. Anthony, J. Appl. Phys., 89, 5243 (2001)

14. R. M. C. de Almeida and I. J. R. Baumvol, Surf. Sci. Rep., 49, 1 (2003).

15. "Impact of fin width on digital and analog performances of nFinFETs," V. Subramanian ,1, Mercha, Parvais, J. Loo. Gustin, M. Dehan, N. Collaert, M. Jurczak, G. Groeseneken 1, W. Sansen 1, S. Decoutere, Solid-State Electronics Volume 51, Issue 4, April 2007, Pages 551-559.

16. M.Balog,M.Schieber, M.Michman and Patai,'The chemical vapour deposition and characterization of $\mathrm{ZrO} 2$ films feom organometallic compounds". Thin Solid films, Volume 47, Issue 2, 1 December 1977, Pages 109-120

17. Balazs Kralik, Eric C. Chang, and steven G.Louice, "Structural properties and quasiparticle band structure of zirconia," physical review B,57(12),pages.7027,1998.

18. Gordon E. Moore, "No Exponential is Forever: But 'Forever' Can Be Delayed." Solid-State Circuits Conference, 2003. Digest of Technical Papers. ISSCC. 2003 IEEE International, 2003, vol.1,pages. 20 - 23

19. Scott E. Thompson, Robert S. Chau, Tahir Ghani, Kaizad Mistry, Sunit Tyagi, and Mark T. Bohr, "In Search of 'Forever,' Continued Transistor Scaling One New Material at a Time," IEEE Transactions on Semiconductor Manufacturing, Vol. 18, No. 1, February 2005, pgs. $26-36$.

20. Woerlee Pierre H, Knitel Mathijs J, van Langevelde Ronald, Klaassen Dirk BM, Tiemeijer Luuk F, Scholten Andries J, et al. TA (2001), "RF-CMOS performance trends.", IEEE Trans Electron Dev, vol. 48, no. 8, pp. 1776-82.

21. Tian $\mathrm{Y}$, Huang $\mathrm{R}$, Zhang $\mathrm{R}$, et al. "A novel nanoscaled device concept: Quasi-SOI MOSFET to eliminate the potential weaknesses of UTB SOI MOSFET," IEEE Trans Elect Dev, 2005, 52(4): $561-568$.

22. E. J. Nowak, "Maintaining the benefits of CMOS scaling when scaling bogs down," Journal of Research and Development, March/May 2002, pgs. $169-18$.

23. T.N. Theis, "Beyond the silicon transistor: personal observations," Computing in Science \& Engineering, Jan.-Feb. 2003, pgs. 25 - 29.

24. T. H. Ning, "Why BiCMOS and SOI BiCMOS?", Journal of Research and Development, March/May 2002, pgs. 181 - 186.

25. X. Huang, W. Lee, C. Kuo, D. Hisamoto, L. Chang, E. Anderson, H. Takeuchi, Y. Choi, K. Asano, V. Subramanian, T. King, J. Bokor, and C. Hu, "Sub-50 nm p-channel FinFET," IEEE Trans. Elelectron Devices, vol. 48, no. 5, pp. 880-884, May 2001.

26. Y. Choi, T. King, and C. Hu, "Nanoscale CMOS spacer FinFET for the terabit era," IEEE Trans. Elelectron Devices, vol. 23, no. 1, pp. 25-27, Jan. 2002.

27. Y.-K. Choi, N. Lindert, P. Xuan, S. Tang, D. Ha, E. Anderson, T.-J. King, J. Bokor, and C. Hu, "Sub-20 nm CMOS FinFET technologies," in IEDM Tech. Dig., 2001, pp. 421-424.

28. Wang YangYuan, Zhang Xing, Liu XiaoYan \& HuanG Ru, “ Novel devices and process for $32 \mathrm{~nm}$ CMOS technology and beyond," Science in China Series F: Information Sciences, Jun. 2008, vol. 51, no. 6, pp. 743-755.

29. Victor V. Zhirnov, Ralph K. Cavin, III, James A. Hutchby, and George I. Bourianoff, "Limits to Binary Logic Switch Scaling-A Gedanken Model," Proceedings of the IEEE, Vol. 91, no. 11, November 2003, pp. 1934 - 1939

30. Hisamoto D, Lee W-C, Kedzierski J, Takeuchi H, Asano K, Kuo C, et al. "FinFET: a self-aligned double-gate MOSFET scalable to 20 nm,”. IEEE Trans Electron Dev 2000; 47(12):2320-5.

31. Lederer D, Kilchytska V, Rudenko T, Collaert N, Flandre D, Dixit A, et al. "FinFET analogue characterization from DC to $110 \mathrm{GHz}$ ". Solid State Electronics, Volume 49, Issue 9, September 2005, Pages 1488-1496.

32. Silicon monolithic integrated circuits in RF systems (SiRF06); 2006. Proceedings,p. 8-11.

33. International Technology Roadmap for Semiconductor 2012.

34. Park J-T, Colinge J-P. "Multiple-gate SOI MOSFETs: device design guidelines" IEEE Trans Electron Device ,2002,volume 49,Issue 12,Pages:2222-2229.

35. Zhang W, Fossum JG, Mathew L, Du Y. "Physical insights regarding design and performance of independent-gate FinFETs,"
IEEE Trans Electron Device 2005;volume 52, Issue 12, pages:2198-2206.

36. Kranti A, Armstrong GA. "Performance assessment of nanoscale double- and triple-gate FinFETs,". Semicond Sci Technol 2006;21:409-21.

37. D. A. Antoniadis, "MOSFET scalability limits and "New frontier" devices," in Proc. Symp. VLSI Technology, 2002, pp. 2-5.

38. T. Ghani, K. Mistry, P. Packan, S. Thompson, M. Stettler, S. Tyagi, and M. Bohr, "Scaling challenges and device design requirements for high performance sub-50 nm gate length planar CMOS transistors," in Proc. Symp. VLSI Technology, 2000, pp. 174-175.

39. L. Chang, S. Tang, T.-J. King, J. Bokor, and C. Hu, "Gate length scaling and threshold voltage control of double-gate MOSFETs," in Int. Electron Device Meet., 2000, pp. 719-722.

40. Daewon Ha, Hideki Takeuchi, Yang-Kyu Choi, and Tsu-Jae King, "Molybdenum Gate Technology for Ultrathin-Body MOSFETs and FinFETs," IEEE transs Electron Devices, dec 2004,vol. . 51, no. 12, pp. 1989-1996.

41. Subramanian V, Mercha A, Parvais B, Loo J, Gustin C, Dehan M, et al. "Impact of fin width on digital and analog performances of nFinFETs, " Solid State Electron 2007; 51:551-9.

42. Shuang-Yuan Chen, Chia-Hao Tu, Jung-Chun Lin, et. al, "On the reverse short-channel effect and threshold voltage roll-off controls for $90 \mathrm{~nm}$ node MOSFETs," Journal of the Chinese Institute of Engineers, 2007, vol. 30, no. 5, pp. 847-853.

43. D.H. Tassis, A. Tsormpatzoglou, C.A. Dimitriadis , G. Ghibaudo, G. Pananakakis, N. Collaert, "Source/drain optimization of underlapped lightly doped nanoscale double-gate MOSFETs, "

Microelectronic Engineering, Nov. 2010, vol. 87, no. 11, pp. 2353 2357.

44. N. Mohankumar, Binit Syamal, C.K. Sarkar, "Investigation of novel attributes of single halo dual-material double gate MOSFETs for analog/RF applications," Microelectronics Reliability 49, pp. 1491-1497, Jul 2009.

45. N. Mohankumar, Binit Syamal, and Chandan Kumar Sarkar, "Influence of channel and gate Engineering on the Analog and RF Performance of DG MOSFETs," IEEE Trans Electron Devices, vol. 57, no. 4, pp. 820-826, Apr 2010.

46. Kranti A, Chung TM, Flandre D, Raskin JP. "Laterally asymmetric channel engineering in fully depleted double gate SOI MOSFETs for high performance analog applications," Solid State Electron 2004; vol 48 :pp. 947-59

47. Pavanello MA, Martino JA, Flandre D. "Analog circuit design using graded channel silicon-on-Insulator n-MOSFETs," Solid State Electron 2002, vol. 46, pp. 1215-25

48. G. Venkateshwar Reddy, M. Jagadesh Kumar, "Investigation of the novel attributes of a single-halo double gate SOI MOSFET: 2D simulation study, " Microelectronics Journal vol 35 pp 761765,July 2004

49. Chakraborty S, Mallik A, Sarkar CK, Ramgopal Rao V. "Impact of halo doping on the subthreshold performance of deep-sub micrometer CMOS devices and circuits for ultralow power analog/mixed signal applications," IEEE Trans Electron Dev 2007;54(2):241-8

50. N. Mohankumar, Binit Syamal, C.K. Sarkar, "Performance and Optimization of Dual Material Gate (DMG) Short Channel BULK MOSFETs for Analog/Mixed Signal Applications, " International Journal of Electronics, Volume 96,Issue 6,First published 2009, Pages $603-611$

51. W. Long, H. Ou, J. Kuo, and K. K. Chin, "Dual-material gate (DMG) field effect transistors," IEEE Trans. Electron Devices, vol. 46, no. 5, pp. 865-870, May 1999.

52. A. Chaudhry and M. J. Kumar, "Investigation of the novel attributes of a fully depleted dual-material gate SOI MOSFET," IEEE Trans. Electron Devices, vol. 51, no. 9, pp. 1463-1467, Sep. 2004.

53. S. Chakraborty, A. Mallik, and C. K. Sarkar, "Subthreshold performance of dual-material gate CMOS devices and circuits for ultralow power analog/mixed-signal applications," IEEE Trans. Electron Devices, vol. 55, no. 3, pp. 827-832, Mar. 2008.

54. J. Yuan and J. C. S.Woo, "A novel split-gate MOSFET design realized by a fully silicided gate process for the improvement of transconductance and output resistance," IEEE Electron Device Lett., vol. 26, no. 11, pp. 829-831, Nov. 2005.

55. X. Zhou, "Exploring the novel characteristics of hetero-material gate field-effect transistors (HMGFETs) with gate-material engineering," IEEE Trans. Electron Devices, vol. 47, no. 1, pp. 113-120, Jan. 2000. 
D.Nirmal, P.Vijaya Kumar, K. Shruti, Binola K Jebalin and N.Mohankumar

/Journal of Engineering Science and Technology Review 7 (2) (2014) 119 - 124

56. Robert Chau, Justin Brask, Suman Datta, Gilbert Dewey, Mark Doczy, Brian Doyle, Jack Kavalieros, Ben Jin, Matthew Metz, Amlan Majumdar, and Marko Radosavljevic, "Application of high$\mathrm{K}$ gate dielectrics and metal gate electrodes to enable silicon and non-silicon logic nanotechnology," June 2005, Vol. 80, no. 1.

57. Rudenko T, Kilchytska V, Collaert N, Jurczak M, Nazarov A, Flandre D. "Reduction of gate-to-channel tunneling current in FinFET structures, "Solid State Electron 2007; 51:1466-72.

58. "Synthesis of $\mathrm{ZrO} 2$ and $\mathrm{TiO} 2$ nanocrystalline powders by hydrothermal process," Yu.V. Kolen'koa, V.D. Maximova, A.A. Burukhinb, V.A. Muhanovb, B.R. Churagulovb. Materials Science and Engineering:C, Volume 23, Issues 6-8, 15 December 2003, Pages 1033-1038.

59. C.R.Manoj and V.Ramgopal Rao, "Impact of High k dielectrics on the device and circuit performance of nanoscale FinFETs," IEEE Electron Device Lett., vol. 28, 2007.

60. Wenjuan Zhu, Jin-Ping Han, Member, IEEE, and T. P. Ma, Fellow, IEEE, "Mobility Measurement and Degradation Mechanisms of MOSFETs Made With Ultrathin High-k Dielectrics," IEEE Trans. Electron Devices, vol. 51, 2004.

61. Kang J F, Yu H Y, Ren C, et al.” Improved electrical and reliability characteristics of $\mathrm{HfN} / \mathrm{HfO}_{2}$ gated nMOSFET with $0.95 \mathrm{~nm}$ EOT fabricated using a gate-first process", IEEE Elect Dev Lett, 2005, 26: $237-239$

62. Nawaz M, Molzer W, Decker S, Giles L-F, Schulz T “ On the device design assessment of multigate FETs (MuGFETs) using full process and device simulation with 3D TCAD., " Microelectronics Journal 2007;38:1238-51.

63. Rudenko T, Kilchytska V, Collaert N, Jurczak M, Nazarov A, Flandre D. "Reduction of gate-to-channel tunneling current in FinFET structures". Solid State Electron 2007; 51:1466-1472.

64. E. J. Nowak, "Maintaining the benefits of CMOS scaling when scaling bogs down," Journal of Research and Development, March/May 2002, pgs. $169-18$

65. T.N. Theis, "Beyond the silicon transistor: personal observations," Computing in Science \& Engineering, Jan.-Feb. 2003, pgs. 25 - 29

66. Kranti A, Armstrong GA. "Performance assessment of nanoscale double- and triple-gate FinFETs" Semicond Sci Technol 2006; 21:409.

67. Park J-T, Colinge J-P. "Multiple-gate SOI MOSFETs: device design guidelines," IEEE Trans Electron Dev 2002; 49:2222-2229.

68. R. M. C. de Almeida and I. J. R. Baumvol, Surf. Sci. Rep.,2003, 49, 1.

69. H. R. Huff, A. Hou, C. Lim, Y. Kim, J. Barnett, G. Bersuker, G. A. Brown, C. D. Young, P. M. Zeitzoff, and J. Gutt, Microelectron. Eng., 69, 152 (2003).

70. J. Robertson, Appl. Surf. Sci., 190, 2 (2002).

71. L. Manchanda, M. D. Morris, M. L. Green, R. B. van Dover, F. Klemens, T. W. Sorsch, P. J. Silverman, G. D. Wilk, B. Busch, and S. Aravamudhan, Microelectron. Eng., 59, 351 (2001).

72. G. D. Wilk, R. M. Wallace, and J. M. Anthony, J. Appl. Phys., 89, 5243 (2001)

73. A. Tsormpatzoglou, C.A. Dimitriadis, M. Mouis, G. Ghibaudo, N. Collaert "Experimental characterization of the subthreshold leakage current in triple-gate FinFETs", Solid-State ElectronicsVolume 53, Issue 3, March 2009, Pages 359-363.

74. k. Fobelets, p.w. Ding, y. Shadrokh j.e. Velazquez-perez. "Analog and digital performance of the screen-grid field effect transistor (SGRFET), “ International Journal of High Speed Electronics and Systems, 2010,Page:1-10.

75. Nirmal D, Nalini B, Vijayakumar P. "Nanosized High k Dielectric Material for FINFET" Integrated Ferroelectrics 2010; 121(1):3135 .

76. Nirmal .D, Vijayakumar .P, Shruthi and Mohan Kumar .N "Nanoscale Channel Engineered Double Gate MOSFET for Mixed Signal Applications using High-k Dielectric", International journal of circuit theory and applications. DOI: $10.1002 /$ cta. 1800

77. Nirmal .D, Vijayakumar .P, Patrick Chella Samuel, Binola k Jebalin, and Mohan Kumar .N "Subthreshold analysis of nanoscale FinFETs for ultra low power application using high-k materials", International Journal of Electronics DOI: $10.1080 / 00207217.2012 .720955$

78. Nirmal .D, Vijayakumar .P, Divya .D, Binola k Jebalin, and Mohan Kumar .N "Subthreshold Performance of Gate Engineered FinFET Devices and circuit with High-k Dielectrics", Microelectronics Reliability. 53 (2013) 499-504.
79. Nirmal .D and Vijayakumar .P "Fin Field Effect Transistors Performance in Analog and RF for High-k Dielectrics" Defence Science Journal, Vol. 61, No. 3, pp. 235-240, May 2011. 